\title{
Driving allotolerance: CAR-expressing Tregs for tolerance induction in organ and stem cell transplantation
}

\author{
Matthias Edinger \\ University Hospital Regensburg, Department of Internal Medicine III (Hematology \& Oncology) and Regensburg Center for Interventional Immunology, Regensburg, Germany.
}

\begin{abstract}
Regulatory T cells (Tregs) modulate the function of a variety of immune cells and are critical for maintaining self-tolerance and preventing the development of autoimmune disease. Due to their ability to suppress effector T cells, Tregs have been increasingly explored for clinical use to suppress alloresponses. While this approach has been promising in preclinical models and early clinical trials, widespread clinical use of Tregs has been limited by the low number of these cells in the periphery and the unknown frequency of allo-responsive Tregs. In this issue of the $J C I$, MacDonald and colleagues transduced human Tregs with a chimeric antigen receptor (CAR) that targets the HLA class I molecule A2. These CARexpressing $T$ cells were readily activated via CAR stimulation and exerted potent immunosuppressive effects when stimulated in vitro. In a murine model of hematopoietic stem cell transplantation, CAR-modified Tregs were more effective in preventing the development of graft-versus-host disease compared with polyclonal Tregs. The results of this study lay the groundwork for the further evaluation of CAR-expressing Tregs in the prevention or treatment of transplant complications.
\end{abstract}

\section{Regulatory T cells for transplant tolerance}

The so-called natural $\mathrm{CD} 4{ }^{+} \mathrm{CD} 25^{+}$regulatory $\mathrm{T}$ cells (Tregs) are thymus-derived suppressor cells that were first characterized in the landmark paper by Shimon Sakaguchi and colleagues as an anergic and suppressive $\mathrm{T}$ cell population that potently inhibits autoimmunity (1). The later discovery that FOXP3 expression determines the thymic development and peripheral function of Tregs (2) justified the designation of this population as a separate $\mathrm{T}$ cell lineage and sparked the interest of the scientific community in Treg biology. Once it was shown that lack-of-function mutations of the FOXP3-encoding gene impair Treg function and cause severe and frequently lethal autoimmunity in mice and humans (scurfy and immune dysregulation, polyendocrinopathy, enteropathy, X-linked [IPEX], respectively) $(3,4)$, it was broadly recognized that Treg-mediated suppression plays a pivotal role in the maintenance of peripheral tolerance. These findings also illustrated that no other regulatory cell population compensates for the lack of FOXP3 ${ }^{+}$ suppressor cells in the periphery. Tregs gain suppressive activity only after stimulation of their own $\mathrm{T}$ cell receptor; however, once activated, Tregs suppress effector $\mathrm{T}$ cells in their vicinity in a nonspecific manner (referred to as bystander or linked suppression). Initially, it was thought that Tregs employ a unique and Treg-specific mode of suppression, but it now seems that these cells apply various and variable suppressive mechanisms in response to

Related Article: p. 1413

Conflict of interest: The author has declared that no conflict of interest exists.

Reference information: / Clin Invest. 2016;126(4):1248-1250. doi:10.1172/JCI86827.

the inflammatory microenvironment (5). Furthermore, Tregs do not solely act on effector $\mathrm{T}$ cells, but modulate the function of virtually every cell type of the innate and adaptive immune system and even act on nonhematopoietic targets (6).

The ability of Tregs to suppress $\mathrm{T}$ cell responses without mediating proinflammatory effects themselves has evoked the interest of transplantation immunologists, as such a cell population may dampen alloresponses without causing tissue damage. In murine disease models, it was shown that the adoptive transfer of donor Tregs prevents severe graft-versus-host disease (GVHD) after allogeneic stem cell transplantation (SCT), which otherwise would be initiated by conventional donor T cells contained in the stem cell graft (7-9). Similarly, host-derived Tregs responding to donor alloantigens have been shown to ameliorate graft rejection after solid organ transplantation, suggesting that strengthening Tregmediated suppression may be an attractive strategy for promoting transplantation tolerance $(10,11)$. Indeed, early clinical trials confirmed the suppressive activity of donor Tregs in SCT, as acute GVHD was prevented in individuals that received haploidentical grafts in the absence of pharmacologic immunosuppression (12).

\section{Applying CAR technology to Tregs}

Second-generation chimeric antigen receptors (CARs), composed of single-chain variable fragments from an antibody fused to costimulatory and TCR signaling endodomains, trigger $\mathrm{T}$ cell responses in an HLAindependent manner upon CAR engagement. CD19-targeted CAR-T cells mediate potent cytotoxicity against malignant (as well as normal) B cells and induce complete remissions in patients with refractory acute lymphoblastic leukemia and certain lymphomas with unprecedented efficacy (13-15). This groundbreaking progress has 
inspired scientists, as well as biotech and pharmaceutical companies, to extend CAR$\mathrm{T}$ cell therapy to other malignant diseases (16). An obvious next step in the exploitation of CAR technologies is the genetic modification of Tregs for the treatment of autoimmune diseases and for the induction of tolerance after allogeneic SCT or solid organ transplantation (17). Initial proof-ofconcept studies have already shown that CAR-redirected Tregs ameliorate experimental colitis (18) and that CAR-modified human Tregs gain suppressive activity upon antigen-specific CAR engagement (19). In this issue, MacDonald and colleagues report on the transduction of human Tregs with a CAR directed against the HLA class I molecule A2 to explore the capacity of these engineered cells to suppress alloresponses in vitro and in xenogenic models in vivo (20). The authors have convincingly shown that lentivirally transduced Tregs stably express the HLA-A2-specific CAR (A2-CAR) without changing their phenotype, FOXP3 expression, or incapacity to secrete proinflammatory cytokines. Upon CAR triggering, Tregs became activated and gained suppressive activity, as illustrated by the inhibition of mixed lymphocyte reactions. MacDonald et al. employed a so-called xenogenic GVHD model to evaluate the efficacy of this approach in vivo. In this model, HLA-A2-positive peripheral blood mononuclear cells (PBMCs), which contain effector $\mathrm{T}$ cells, are infused into immunodeficient NOD/SCID IL-2R $\gamma^{\text {null }}$ (NSG) mice and induce lethal disease. Cotransfer of anti-A2-CAR-modified Tregs ameliorated the development of lethal GVHD in this model, while cotransfer of Tregs expressing a HER2-specific control CAR did not improve outcome. Moreover, immune-monitoring data suggest that the observed delay in disease onset is caused by the Treg-mediated inhibition of effector T cell engraftment and expansion. Even though this kind of experiment is often performed (and frequently requested by reviewers) to illustrate human Treg efficacy for the prevention of GVHD, results should be interpreted with caution, as the xenogenic disease has hardly anything in common with human GVHD or GVHD in allogeneic transplant models. Furthermore, human Tregs do not survive in such hosts for more than a few days and thus can only act during the disease initiation phase, but not for the long term. Hence, such models can be confirmative at most (in cases where the expected results are observed), but are unsuited to study the basic biology of human Tregs in vivo. The same considerations also apply to safety aspects of CARmodified Treg therapy that were addressed by MacDonald et al. in supplemental experiments in which HLA-A2-transgenic NSG mice were used as hosts. The lack of tissue damage after transfer of A2-CAR Tregs is reassuring, yet it should be noted that these immunodeficient xenogenic recipients lack pivotal cells and effector mechanisms/molecules that may indirectly contribute to tissue damage upon recognition of HLA-A2 on target organs by CAR-modified Tregs. Despite these reservations, the use of CARmodified Tregs is an attractive new strategy for enhancing transplantation tolerance in the future, and these Tregs are well worth examining in more detail in appropriate experimental models in preparation for first clinical trials. Several issues, including the selection of the CAR target antigens, remain to be addressed in more pertinent models. Tregs are $\mathrm{CD} 4^{+} \mathrm{T}$ cells that are usually restricted by HLA class II molecules. In murine models, the lymph node-homing capacity of donor Tregs is required for protection from acute GVHD, suggesting that class II-positive professional antigenpresenting cells (APCs) stimulate alloreactive Tregs and license them for the suppression of alloreactive effector $\mathrm{T}$ cells in lymphoid organs (21). Yet for therapeutic purposes, it may be that Treg-mediated suppression is primarily required in target organs and that activation via locally expressed HLA class I molecules is beneficial. However, the ubiquitous expression of class I molecules increases the risk of generalized immunosuppression by class I-targeted CAR-expressing Tregs and therefore might be detrimental for SCT patients with residual leukemia or lymphoma who rely on efficient graft-versus-leukemia effects and are prone to opportunistic infections. The incorporation of suicide genes or molecular on/off switches into CAR-expressing Tregs could enhance the safety profile of such suppressor cell products (22).

\section{Clinical translation}

The main hurdles for the clinical translation of experimental Treg therapies have been the paucity of thymus-derived
Tregs in peripheral blood and the lack of specific isolation and efficient in vitro expansion protocols. MacDonald and colleagues have now isolated CD45RA ${ }^{+}$ Tregs, a strategy that diminishes the risk of contamination with conventional $\mathrm{T}$ cells and ensures improved Treg lineage stability upon in vitro stimulation and expansion $(23,24)$. The success of this strategy was verified by the analysis of epigenetic signatures of Treg stability, such as demethylation of the Treg-specific demethylation region (TSDR) within the FOXP3 locus, a prerequisite for stable FOXP3 expression (25). Nevertheless, the ability to isolate human Tregs from peripheral blood without contaminating effector $\mathrm{T}$ cells is still a challenge, and good-manufacturing-practice-compliant (GMP-compliant) technologies for the isolation of Treg subpopulations are not yet generally available. However, new strategies that permit reversible labeling for cell enrichment are currently being developed and might soon permit the stepwise purification of Treg subpopulations (26). Furthermore, our group, as well as a few other groups, has recently established GMP-compatible flow cytometric sort technologies for clinical applications, and some companies have now developed optimized high-speed cell sorters to provide flexible tools for the purification of rare $\mathrm{T}$ cell subpopulations from peripheral blood. Thus, CARs have not only advanced genetic $\mathrm{T}$ cell modification technologies, but have also encouraged technology suppliers to invest in new $\mathrm{T}$ cell isolation and expansion reagents. In our hands, FACSpurified CD45RA ${ }^{+}$Tregs maintain all the phenotypic and functional characteristics of thymic Tregs after isolation and extended in vitro expansion. Moreover, such cell products increased the Treg pool of several patients treated for refractory acute GVHD within an ongoing clinical trial at our institution (EudraCT 2012-002685-12). Redirecting Tregs toward a single HLA mismatch by CARs is a promising next step in the exploration of Treg-mediated suppression of alloresponses because bystander suppression may be sufficient to prevent (and eventually suppress an ongoing) polyclonal $\mathrm{T}$ cell response after HLA-mismatched stem cell or solid organ transplanta- 
tion. However, more work will need to be done, including a better evaluation of CAR-modified Tregs in preclinical models, before this treatment option can be applied to patients.

\section{Acknowledgments}

M. Edinger gratefully acknowledges research funding support by the German Research Foundation (KFO146), the European Commission (Horizon 2020), the José Carreras Leukemia Foundation, the Wilhelm Sander Foundation, and the Free State of Bavaria (BayImmuNet, RCI).

Address correspondence to: Matthias Edinger, University Hospital Regensburg, Department of Internal Medicine III (Hematology \& Oncology) and Regensburg Center for Interventional Immunology, Franz-JosefStrauss-Allee 11, 93053 Regensburg, Germany. Phone: 49.0.941.944.5580; E-mail: matthias.edinger@ukr.de.

1. Asano M, Toda M, Sakaguchi N, Sakaguchi S. Autoimmune disease as a consequence of developmental abnormality of a $\mathrm{T}$ cell subpopulation. J Exp Med. 1996;184(2):387-396.

2. Fontenot JD, Gavin MA, Rudensky AY. Foxp3 programs the development and function of $\mathrm{CD} 4{ }^{+} \mathrm{CD} 25^{+}$regulatory T cells. Nat Immunol. 2003;4(4):330-336.

3. Bennett CL, et al. The immune dysregulation, polyendocrinopathy, enteropathy, X-linked syndrome (IPEX) is caused by mutations of FOXP3. Nat Genet. 2001;27(1):20-21.

4. Brunkow ME, et al. Disruption of a new fork- head/winged-helix protein, scurfin, results in the fatal lymphoproliferative disorder of the scurfy mouse. Nat Genet. 2001;27(1):68-73.

5. Chaudhry A, Rudensky AY. Control of inflammation by integration of environmental cues by regulatory T cells. JClin Invest. 2013;123(3):939-944.

6. Josefowicz SZ, Lu LF, Rudensky AY. Regulatory T cells: mechanisms of differentiation and function. Annu Rev Immunol. 2012;30:531-564.

7. Hoffmann P, Ermann J, Edinger M, Fathman CG, Strober S. Donor-type CD4(+)CD25(+) regulatory $\mathrm{T}$ cells suppress lethal acute graft-versus-host disease after allogeneic bone marrow transplantation. J Exp Med. 2002;196(3):389-399.

8. Taylor PA, Lees CJ, Blazar BR. The infusion of ex vivo activated and expanded CD4(+)CD25(+) immune regulatory cells inhibits graft-versus-host disease lethality. Blood. 2002;99(10):3493-3499.

9. Cohen JL, Trenado A, Vasey D, Klatzmann D, Salomon BL. CD4(+)CD25(+) immunoregulatory T cells: new therapeutics for graft-versushost disease. JExp Med. 2002;196(3):401-406.

10. Joffre $\mathrm{O}$, et al. Prevention of acute and chronic allograft rejection with $\mathrm{CD} 4{ }^{+} \mathrm{CD} 25^{+} \mathrm{Foxp}^{+}$regulatory T lymphocytes. Nat Med. 2008;14(1):88-92.

11. Waldmann H, Hilbrands R, Howie D, Cobbold S. Harnessing FOXP3 ${ }^{+}$regulatory T cells for transplantation tolerance. J Clin Invest. 2014;124(4):1439-1445.

12. Di Ianni M, et al. Tregs prevent GVHD and promote immune reconstitution in HLA-haploidentical transplantation. Blood. 2011;117(14):3921-3928.

13. Davila ML, et al. Efficacy and toxicity management of 19-28z CAR T cell therapy in B cell acute lymphoblastic leukemia. Sci Transl Med. 2014;6(224):224ra225.

14. Lee DW, et al. T cells expressing CD19 chimeric antigen receptors for acute lymphoblastic leukaemia in children and young adults: a phase 1 dose-escalation trial. Lancet. 2015;385(9967):517-528.

15. Maude SL, et al. Chimeric antigen receptor T cells for sustained remissions in leukemia. N Engl J Med. 2014;371(16):1507-1517.

16. June $\mathrm{CH}$, Riddell SR, Schumacher TN. Adoptive cellular therapy: a race to the finish line. $\mathrm{Sci}$ Transl Med.2015;7(280):280ps287.

17. Jethwa H, Adami AA, Maher J. Use of gene-modified regulatory T-cells to control autoimmune and alloimmune pathology: is now the right time? Clin Immunol. 2014;150(1):51-63.

18. Elinav E, Adam N, Waks T, Eshhar Z. Amelioration of colitis by genetically engineered murine regulatory $\mathrm{T}$ cells redirected by antigenspecific chimeric receptor. Gastroenterology. 2009;136(5):1721-1731.

19. Hombach AA, Kofler D, Rappl G, Abken H. Redirecting human $\mathrm{CD} 4{ }^{+} \mathrm{CD} 25^{+}$regulatory T cells from the peripheral blood with pre-defined target specificity. Gene Ther. 2009;16(9):1088-1096.

20. MacDonald KG, et al. Alloantigen-specific regulatory $\mathrm{T}$ cells generated with a chimeric antigen receptor. JClin Invest. 2016;126(4):1413-1424.

21. Ermann J, et al. Only the $\mathrm{CD} 62 \mathrm{~L}^{+}$subpopulation of $\mathrm{CD} 4{ }^{+} \mathrm{CD} 25^{+}$regulatory $\mathrm{T}$ cells protects from lethal acute GVHD. Blood. 2005;105(5):2220-2226.

22. Rodgers DT, et al. Switch-mediated activation and retargeting of CAR-T cells for B-cell malignancies. Proc Natl Acad Sci U S A. 2016;113(4):E459-468.

23. Hoffmann P, et al. Only the CD45RA ${ }^{+}$subpopulation of $\mathrm{CD} 4^{+} \mathrm{CD} 25^{\text {high }} \mathrm{T}$ cells gives rise to homogeneous regulatory $\mathrm{T}$-cell lines upon in vitro expansion. Blood. 2006;108(13):4260-4267.

24. Hoffmann P, et al. Loss of FOXP3 expression in natural human $\mathrm{CD} 4{ }^{+} \mathrm{CD} 25^{+}$regulatory T cells upon repetitive in vitro stimulation. Eur J Immunol. 2009;39(4):1088-1097.

25. Huehn J, Polansky JK, Hamann A. Epigenetic control of FOXP3 expression: the key to a stable regulatory T-cell lineage? Nat Rev Immunol. 2009;9(2):83-89.

26. Stemberger C, et al. Novel serial positive enrichment technology enables clinical multiparameter cell sorting. PLoS One. 2012;7(4):e35798. 